\title{
Editorial: Intertemporal Choice and Its Anomalies
}

\author{
Salvador Cruz Rambaud ${ }^{1 *}$ and Taiki Takahashi ${ }^{2}$ \\ ${ }^{1}$ Departamento de Economía y Empresa, University of Almería, Almería, Spain, ${ }^{2}$ Department of Behavioral Science, \\ Hokkaido University, Sapporo, Japan
}

Keywords: intertemporal choice, anomaly, behavioral economics, time preference, time-consistency

\section{Editorial on the Research Topic}

\section{Intertemporal Choice and Its Anomalies}

This special issue on "Intertemporal choice and its anomalies" collected up-to-date papers on the topic from various research disciplines: behavioral economics, computer science, mathematical, cognitive and cultural psychology, behavioral ecology, and econophysics. This issue demonstrates that the studies of intertemporal choice and its anomalies have been extended into important research activities outside of traditional domains of economics.

In economics, intertemporal choice has been modeled with the concept of time preference, and rationality (i.e., time-consistency) in intertemporal choice has also been assumed. This conceptualization was shown to be useful to investigate a wide variety of social problems such as addiction, retirement plans, and health-maintaining behaviors even when the violation of the time-inconsistency was not taken into account. Recent developments in behavioral economics suggest that once irrationality in intertemporal choice (anomalies such as hyperbolic discounting or delay effect, sign effect, magnitude effect, delay-speedup asymmetry, and sequence effects) were seriously considered, many problematic behaviors can be considered as manifestations of self-control problems in intertemporal choice.

This issue demonstrates that behavioral economic approaches to self-control problems in intertemporal choice have been attracting wide attention from diverse disciplines of science. The editors are pleased to witness this spread of interest into broad research areas and we hope the movement speed up without temporal discounting over time.

Ogura et al. introduce a model in the ambit of foraging ecology which predicts that the profitability of a smaller-sooner (SS) food option can be higher than that of the larger-later (LL) alternative, depending on the duration in which the producer can monopolize a food patch. Their paper includes numerical simulations on the assumption of variable food amount in each patch involving realistic behavioral parameters.

Cruz Rambaud and Sánchez Pérez reveal the existence of several anomalies or paradoxes in the context of EU and DU models affecting the amount of the reward: the peanuts and the magnitude effects, respectively, which seem go in opposite directions. Their paper jointly analyses both effects in a wide setting involving choices under risk and over time, searching implications between both anomalies.

On the other hand, Cruz Rambaud et al. formalize mathematically the concept of improving sequence effect. More specifically, they prove that the improving sequence effect for monetary rewards must be necessarily rationalized by using a non-separable discount function. Moreover, under certain conditions, they demonstrate that the delay and the magnitude effects are necessary (but not sufficient) conditions for the existence of the improving sequence effect.

Tsuruta and Inukai aim to assess the role of group intertemporal decision-making. They experimentally investigate how to aggregate individual time preferences by clarifying who has the most influence on group decisions among heterogeneous group members. They formulate two hypotheses: the multilateral bargaining hypothesis, which is based on the multilateral bargaining model, and the median voter hypothesis. 
The main contribution of this paper is that the median patient member in a group has a significant impact on group decisions in an unstructured bargaining situation.

dos Santos and Martinez derive inconsistency as the result of a subjective time dilation perception effect, inspired by the special theory of relativity. They focus on a generalized model which encompasses psychophysical effects on time perception, by proposing a transformation of the time interval between the pay times of two rewards. As a result, they present a generalized two-argument hyperbolic utility function for the Bernoulli (logarithmic) one.

Takemura and Murakami present a test to examine various models of probability weighting functions which are considered non-linear functions of probability in behavioral decision theory. On the other hand, they propose some axiomatic properties and a test to specifically examine the generalized hyperbolic logarithmic model, power model, and exponential power model of the probability weighting functions.

Kim and Takahashi state that, in computational theory of learning, the multi-armed bandit problem is one of the most intensively studied decision problems with unknown rewards and probability distributions. In economic decision theory, two types of uncertainty, i.e., risk and ambiguity (also referred to as Knightian uncertainty) have been distinctly formulated. Risk is uncertainty with known probability distributions, while ambiguity is uncertainty with unknown distributions. The latter is closely linked with the multi-armed bandit problem. In the study, we employed several state-of-the-art computational learning algorithms to resolve the optimization problem of decision under ambiguity. This computational approach may be unified with behavioral economic approaches to ambiguity problem in the future.

Ishii and Eisen start from the idea of social discounting. They carry out two studies. The first one showed that compared to North Americans, Japanese discount more steeply a partner's outcomes compared to their own future outcomes, whilst the decrease in the subjective value of the partner's outcomes accelerates less as a function of social distance. The second study tested Japanese and Germans and found that the hyperbolic with exponent model fitted the participants' discounting behaviors better than the other models.
Attema and Lipman implement a recently introduced measure of deviations from constant impatience, called the "Decreasing Impatience (DI)-index," to estimate the degree to which people deviate from constant impatience. They observe that decreasing impatience is the modal preference, although constant and increasing impatience are no exceptions. Furthermore, the DIindex is higher for individual health outcomes than for societal health outcomes, but is not distributed differently among the three classes of discounters.

Li et al. analyse how future time perspective (FTP) affects intertemporal choice. To do this, the conducted an experiment where all participants completed a series of intertemporal choice tasks, in which they chose from gain- and loss-related choices occurring at various time points. Results showed that the participants who received the future-imagining manipulation had more limited FTP. The participants in the limited FTP condition had higher discount rates on gain-related choices but showed no difference on loss-related choices.

\section{AUTHOR CONTRIBUTIONS}

All authors listed have made a substantial, direct and intellectual contribution to the work, and approved it for publication.

\section{FUNDING}

This paper has been partially supported by the project La Sostenibilidad del Sistema Nacional de Salud: reformas, estrategias y propuestas, reference: DER2016-76053-R, Ministerio de Economía y Competitividad.

Conflict of Interest Statement: The authors declare that the research was conducted in the absence of any commercial or financial relationships that could be construed as a potential conflict of interest.

Copyright (c) 2019 Cruz Rambaud and Takahashi. This is an open-access article distributed under the terms of the Creative Commons Attribution License (CC BY). The use, distribution or reproduction in other forums is permitted, provided the original author(s) and the copyright owner(s) are credited and that the original publication in this journal is cited, in accordance with accepted academic practice. No use, distribution or reproduction is permitted which does not comply with these terms. 